\title{
Two-photon optical-beam-induced current imaging of indium gallium nitride blue light-emitting diodes
}

\author{
Fu-Jen Kao, Mao-Kuo Huang, and Yung-Shun Wang \\ Department of Physics, National Sun Yat-Sen University, Kaohsiung 80424, Taiwan \\ Sheng-Lung Huang \\ Institute of Electro-Optical Engineering, National Sun Yat-Sen University, Kaohsiung 80424, Taiwan \\ Ming-Kwei Lee \\ Department of Electrical Engineering, National Sun Yat-Sen University, Kaohsiung 80424, Taiwan \\ Chi-Kuang Sun \\ Institute of Electro-Optical Engineering, National Taiwan University, Taipei 106, Taiwan
}

Received May 17, 1999

\begin{abstract}
Epilayers of packaged indium gallium nitride light-emitting diodes (LED's) are characterized by optical-beaminduced current (OBIC) and photoluminescence laser-scanning microscopy through two-photon excitation. Light scattering and absorption in the packaging material and the $p$-doped top layer of the LED's are greatly reduced as a result of employing a longer excitation wavelength, with energy that is less than the bandgap of the top $p$ layer. Compared with single-photon OBIC, two-photon OBIC imaging not only exhibits superior image quality but also reveals more clearly the characteristics of the epilayers that are being focused on. (c) 1999 Optical Society of America

OCIS codes: $180.1790,230.3670,250.5230,260.5150$.
\end{abstract}

Optical-beam-induced current (OBIC) imaging is widely applied in the characterization of semiconductor-based devices and integrated circuits, for instance, in failure analysis. ${ }^{1,2}$ Conventionally, OBIC is performed through carrier generation by single-photon absorption. However, OBIC imaging of semiconductor devices has to meet the seemingly contradictory conditions that (a) the substrate or overlayers do not absorb or scatter the illuminating light strongly and (b) the photoexcited carriers are efficiently generated in the active layer. These requirements can be met if OBIC imaging is performed with two-photon excitation $^{3}$ that employs wavelengths that are less than the bandgap photon energy, as demonstrated by $\mathrm{Xu}$ and Denk. ${ }^{4}$

In this Letter we apply the two-photon OBIC technique to investigate indium gallium nitride- $(\mathrm{InGaN}-)$ based light-emitting diodes (LED's), which have found widespread applications in optoelectronics. ${ }^{5}$ InGaN is considered to be the most important compound semiconductor among III-V nitride compounds because it permits light emission through efficient carrier recombination. ${ }^{6}$ One can obtain strong band-to-band emission from the green to the UV by varying the In content of InGaN. The emission is related to the presence of deep localized energy states that may originate from the In-rich regions, which act as quantum dots. ${ }^{7-11}$ However, the intensity distribution of LED's can exhibit poor spatial uniformity in the active region, as can be seen with a microscope when LED's are forward biased. This poor spatial uniformity is associated with the fluctuation in In concentration and with localized defects such as deficiency of nitrogen atoms in the lattice and threading dislocations. ${ }^{6}$ We have found that two-photon excitation is effective in penetrating the packaging as well as the overlayers, such as $p$-doped GaN layers or an $n$-doped GaN layer and a sapphire substrate, if the laser beam is incident from the substrate side. Compared with the loss in single-photon excitation, the loss in two-photon excitation that is due to absorption and scattering is greatly reduced. As a result, two-photon OBIC imaging not only exhibits superior image quality but also reveals more clearly the characteristics of the epilayers that are focused on.

An inverted microscope and a galvano-mirror-based scanning system (Fluoview-IX70, Olympus) are employed as the imaging platforms. A mode-locked Ti:sapphire pulse laser (Tsunami, Spectra-Physics) pumped by a frequency-doubled solid-state laser (Millennia, Spectra-Physics) provides laser pulses of approximately $150 \mathrm{fs}$ at $760 \mathrm{~nm}$ for two-photon excitation, which is equivalent to $380 \mathrm{~nm}$ in excitation energy. The average power that is incident upon the sample is 5 to $10 \mathrm{~mW}$. A $40 \times$ (numerical aperture, $0.65)$ long-working-distance objective is employed for image acquisition. The diode samples are also commercially available and have electroluminescence that peaks at $475 \mathrm{~nm}$, with a FWHM bandwidth of $35 \mathrm{~nm}$. Thinning, grinding, and polishing the packaging of the diode allow observation and excitation under a microscope. We employ bandpass filters to select specific spectral bands for luminescence imaging. We employ a very sensitive current amplifier (EG\&G Model 181) with transimpedance as high as $10^{9} \mathrm{~V} / \mathrm{A}$ to detect 
the photocurrent (PC). The detected signal would correspond to a photocurrent of a few nanoamperes. The two input channels in the scanning system detect the photoluminescence (PL) and the PC concurrently. A schematic of this setup is shown in Fig. 1.

Figures 2(a) and 2(b) show the power and the depth dependence of the two-photon PC signal, respectively. As expected for two-photon excitation, the slope value of 1.9 in Fig. 2(a) indicates the square dependence of such a nonlinear process. Figure 2(b) records the PC signal as the LED's are moved across the focal position vertically. The signal drops drastically as soon as the focal plane is moved out of the active region of the LED's. However, the asymmetry of the curve indicates the existence of spherical aberration ${ }^{12}$ inside the LED's. The spherical aberration is expected, since the thinned packaging $(n \sim 1.5)$ on top of the active layers has a thickness of $\sim 0.5 \mathrm{~mm}$ and there is substantial index mismatch with air $(n \sim 1.0)$.

Two-photon PC and PL images of InGaN LED's are presented in Figs. 3(a) and 3(b), respectively. The two electrodes are clearly shown in Fig. 3(a) as one circular and one square dark region since there are no carriers generated underneath. The thin metallization layer is identified as the darker gray area covering most of the active region shown in the PC image, whereas the bright strip in the edge shows the active region without thin metallization. The mostpronounced features in the PC image are the bright spots covering the LED's, which are a result of local variation in carrier transportation or generation efficiency. According to Nakamura and co-workers, ${ }^{7-11}$ fluctuation in the In concentration will generate localized deep levels that greatly facilitate carrier recombination, and possibly carrier generation. These spots have sizes ranging from $5 \mu \mathrm{m}$ to less than the resolution of the objective used in our optical system, which is approximately $1.3 \mu \mathrm{m}$. The spots are similar to what has been observed in microspectroscopy of cathodoluminescence in InGaN quantum wells. ${ }^{11}$ Spots can also be found in the PL image, which was acquired with a bandpass filter of central wavelength $450 \mathrm{~nm}$ and bandwidth $50 \mathrm{~nm}$. These spots overlap those found in the PC image, indicating that PC and PL have a similar origin. However, the contrast in the PL image is less pronounced. Therefore PC may be a more sensitive indicator than PL in detecting local variation in the active region. The advantage of two-photon excitation is further clarified by comparison with the PC image obtained through single-photon excitation, shown in Fig. 3(c). The 488-nm line of an argon krypton mixed-gas laser is employed for excitation. The image appears blurred with reduced contrast, and no detailed features can be observed. This blurring is attributed to spherical aberration owing to index mismatch and cannot be corrected simply by adjustment of the focal plane. ${ }^{12}$

Efficient light emission of a blue LED is related to deep localized energy states formed in the In-rich regions, which act as quantum dots in the InGaN layer. These states may also be the origin of the PC. Since the direct bandgap $(\sim 3.4 \mathrm{eV})$ of intrinsic $\mathrm{GaN}$ is much higher than that of InGaN, it is less probable that the wavelength that we used would excite carriers over GaN's bandgap. According to Nakamura and Fasol, ${ }^{5}$ although the PL spectra of $p$-doped GaN and InGaN

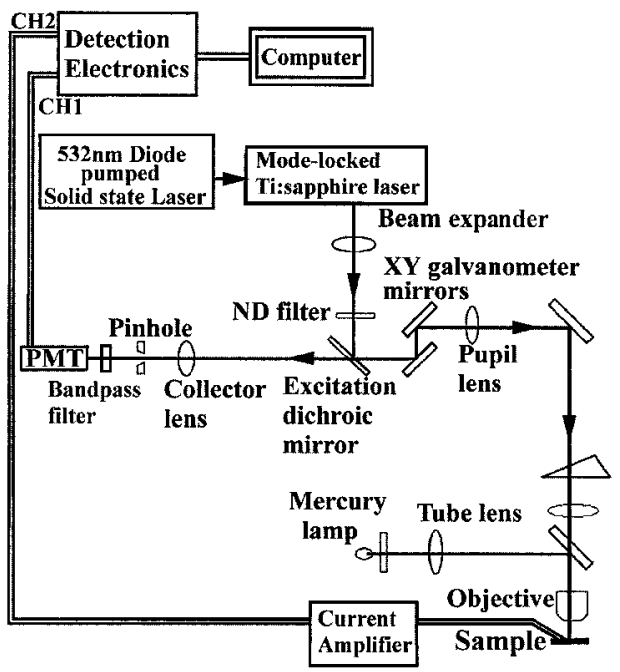

Fig. 1. Schematic of the two-photon OBIC and PL imaging system. The two input channels $(\mathrm{CH} 1, \mathrm{CH} 2)$ allow simultaneous acquisition of PC and PL images. ND filter, neutral-density filter; PMT, photomultiplier tube.

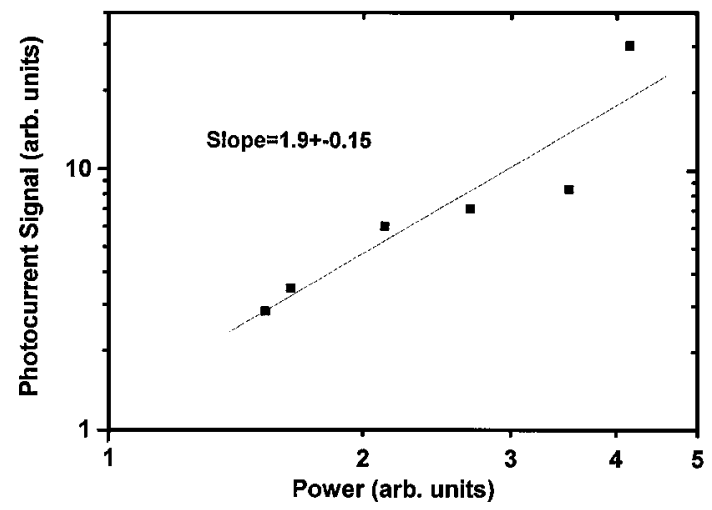

(a)

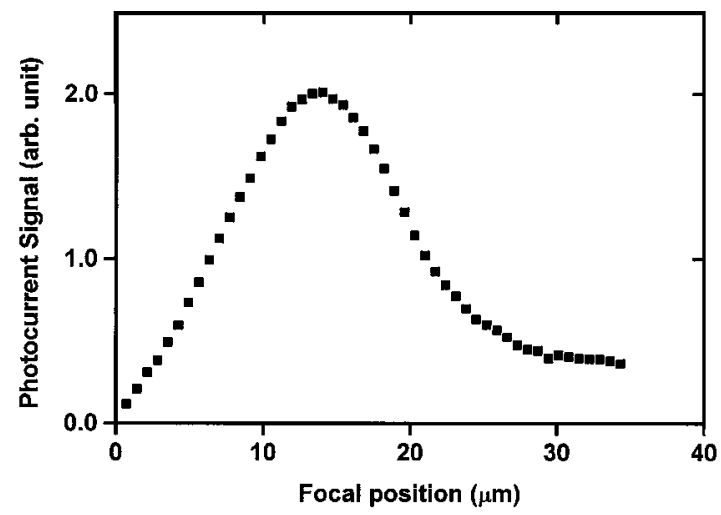

(b)

Fig. 2. (a) Two-photon photoncurrent as a function of incident power. The slope of 1.9 indicates the nonlinear nature of the two-photon process. (b) Depth dependence of the two-photon photocurrent. The focal position corresponds to the actual location of the objective. The asymmetry of this curve indicates the effect of spherical aberration. 


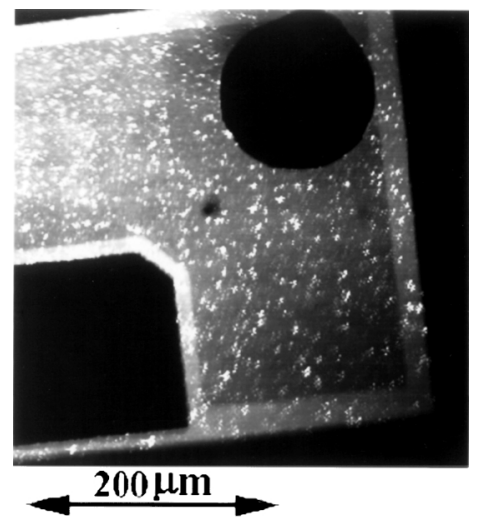

(a)

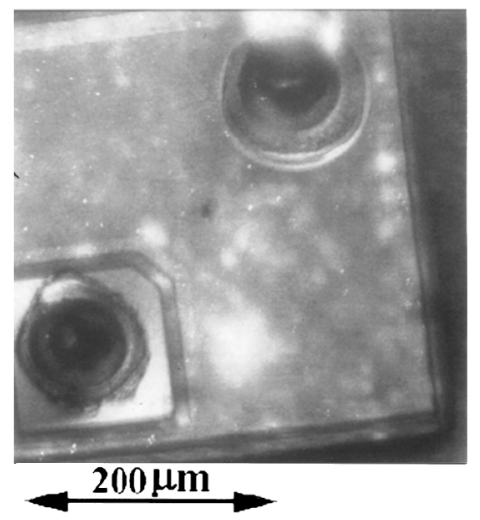

(b)

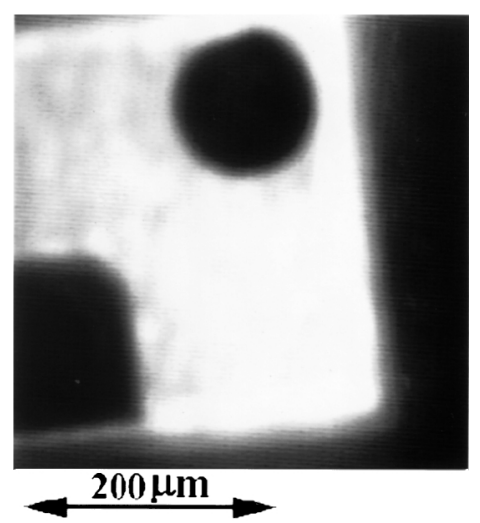

(c)

Fig. 3. Two-photon photocurrent image of an InGaNbased LED. All images are $1024 \times 1024$ pixels. The smallest feature that can be resolved is approximately $1.3 \mu \mathrm{m}$ in size. (b) The corresponding two-photon PL image acquired simultaneously with (a). A combination of a cold mirror and a bandpass filter with central wavelength $450 \mathrm{~nm}$ and FWHM bandwidth $50 \mathrm{~nm}$ is used to reject background. Since the active layer is very thin $(\sim 0.15 \mu \mathrm{m})$, nonconfocal detection is used. (c) Singlephoton photocurrent image of the same LED. The 488-nm line from an argon-krypton laser was employed for excitation. The image blurring cannot be corrected by adjustment of the height of the focal plane, which is an indication of spherical aberration.

overlap, the PL intensity from InGaN is higher by more than an order of magnitude. It is likely that most photogenerated carriers are generated in the InGaN layer.
In OBIC the detected signal can be regarded as the convolution of carrier generation and transportation to the electrodes. Owing to the LED's planar structure and the relatively low resistance in the $p$-doped and $n$-doped layers, the overall carrier transportation efficiency should present little variation over the LED plane. Therefore the spots in Fig. 3(a) may reflect local variation of carrier generation efficiency in the active layer. This variation may also be caused by changes in In compositions. The higher contrast in the PC image than in the PL image indicates that PC is more sensitive to local variation in carrier generation.

In conclusion, we have characterized InGaN-based LED's through two-photon OBIC laser-scanning microscopy. Compared with single-photon excitation, two-photon excitation clearly exhibits better spatial resolution and reveals interesting features that, to our knowledge, were not found before. Improvements in spatial resolution are attributed to much less scattering and absorption of two-photon excitation. ${ }^{13}$ Optoelectronic devices have a multilayered heterostructure, with active layers buried in the middle. Two-photon excitation is more effective than singlephoton excitation in reaching the active layers of these devices. Specifically, the PC is more sensitive than $\mathrm{PL}$ in showing their characteristics.

We gratefully acknowledge support of this research by National Science Council of Taiwan Grants NSC88-2112-M-110 and NSC-88-2216-E-110. F.-J. Kao's e-mail address is fjk@mail.nsysu.edu.tw.

\section{References}

1. T. Wilson and C. J. R. Sheppard, Theory and Practice of Scanning Optical Microscopy (Academic, New York, 1984).

2. B. P. Richards and P. K. Footner, The Role of Microscopy in Semiconductor Failure Analysis (Oxford U. Press, New York, 1992).

3. W. Denk, J. H. Strickler, and W. W. Webb, Science 248, 73 (1990).

4. C. Xu and W. Denk, Appl. Phys. Lett. 71, 2578 (1997).

5. S. Nakamura and G. Fasol, The Blue Laser Diode (Springer-Verlag, Berlin, 1997).

6. S. Nakamura, Science 281, 956 (1998).

7. S. Chichibu, T. Azuhata, T. Sota, and S. Nakamura, Appl. Phys. Lett. 69, 4188 (1997).

8. S. Chichibu, T. Azuhata, T. Sota, and S. Nakamura, Appl. Phys. Lett. 70, 2822 (1997).

9. Y. Narukawa, Y. Kawakami, M. Funato, Sz. Fujita, Sg. Fujita, and S. Nakamura, Appl. Phys. Lett. 70, 981 (1997).

10. Y. Narukawa, Y. Kawakami, Sz. Fujita, Sg. Fujita, and S. Nakamura, Phys. Rev. B 55, 1938R (1997).

11. S. Chichibu, K. Wada, and S. Nakamura, Appl. Phys. Lett. 71, 2346 (1997).

12. M. Gu, Three-Dimensional Confocal Microscopy (World Scientific, Singapore, 1996).

13. D. Day and M. Gu, Appl. Opt. 37, 6299 (1998).

14. P. Török, P. Varga, Z. Laczik, and G. R. Booker, J. Opt. Soc. Am. A 12, 325 (1995).

15. S. P. Schilders, "Microscopic imaging in turbid media," Ph.D. dissertation (Victoria University, Melbourne, Australia, 1999). 